\title{
Design Challenges of Wellbeing Supporting Smart Environment in Collaborative Use Situations
}

\author{
Minna Pakanen ${ }^{182}$, Lauri Lovén ${ }^{1}$, Paula Alavesa ${ }^{1}$, Ekaterina \\ Gilman 1, Jacques Terken ${ }^{3}$, Berry Eggen ${ }^{3}$, and Susanna \\ Pirttikangas ${ }^{1}$ \\ ${ }^{1}$ Center for Ubiquitous Computing, \\ ${ }^{2}$ INTERACT Research Unit, \\ 1 P.O Box 4500, 2 P.O Box 8000 \\ FI-90014 University of Oulu, \\ Finland \\ firstname.lastname@oulu.fi \\ ${ }^{3}$ Department of Industrial Design \\ Eindhoven University of Technology \\ P.O Box 513 \\ $5600 \mathrm{MB}$ Eindhoven \\ The Netherlands \\ $\{j . m . b . t e r k e n ; j . h . e g g e n\} @ t u e . n l$ \\ Permission to make digital or hard copies of all or part of this work for \\ personal or classroom use is granted without fee provided that copies are \\ not made or distributed for profit or commercial advantage and that \\ copies bear this notice and the full citation on the first page. Copyrights \\ for components of this work owned by others than ACM must be honored. \\ Abstracting with credit is permitted. To copy otherwise, or republish, to \\ post on servers or to redistribute to lists, requires prior specific \\ permission and/or a fee. Request permissions from \\ Permissions@acm.org.
}

UbiComp/ISWC'18 Adjunct, October 8-12, 2018, Singapore, Singapore (c) 2018 Association for Computing Machinery.

ACM ISBN 978-1-4503-5966-5/18/10..\$15.00

https://doi.org/10.1145/3267305.3267691

\section{Abstract}

Recent technological development offers new

possibilities for taking into account peoples' personal wellness data in adjustment of environment conditions. For example, users' heartrate, facial expression, room temperature, and $\mathrm{CO}^{2}$ data could be used for adjustment of lighting, temperature, and air-condition to support people's wellbeing in smart environment.

Using personal wellness data for adaptive smart environment condition controlling seems inviting, however before expensive implementation is started, it is essential to know peoples' perceptions towards such systems to be able to create environments that people want to use. We conducted an anticipated user experience study of an adaptive wellbeing supporting meeting room concept video with 48 participants. Based on our results we present the four design challenges for future research.

\section{Author Keywords}

Anticipated user experience; Wellbeing; Smart

environment; User study.

\section{ACM Classification Keywords}

H.5.m. Information interfaces and presentation (e.g., $\mathrm{HCI}$ ): Miscellaneous. 


\section{System input:}

Wearable sensors (a): rings/bracelets (heart rate, stress level), 360 degree camera with facial recognition SW (b) (facial expressions, e.g., emotions), and built-in sensors (b) $\left(\mathrm{CO}^{2}\right.$ level and temperature).

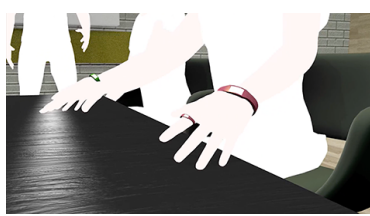

b)

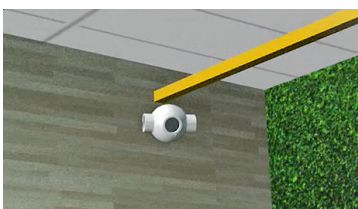

Figure 1: Wearable sensors (a). Build-in sensors and 360 degree camera with facial recognition software (b).

\section{What system does:}

System analyses sensor and camera data collected from the users and the room. Based on this, the system adjusts lighting and airconditioning of the room.

\section{Introduction}

When technology disappears into our surroundings 15], the space can be called as a smart environment. Smart environment rely on real world sensory data used for anticipating human actions and needs with the aim to adapt and automate premise controls to make peoples' experiences of that environment better $[3,5]$ Prior research has suggested topics and challenges for future smart environment research. Such as, an effective use of smart spaces with automatic adjustment of room conditions based on user profiles and invisibility, e.g., minimal user distraction by space meeting the needs of the user seamlessly [14].

Challenges and problems may arise when a space has multiple users instead of one and especially when users have conflicting goals in the space [3]. Using a space as a mechanism to influence individuals' activity patterns, mood, mind, and state of health has been suggested for future investigations, however, this raises several challenges with user privacy [3]. Based on the previous findings, it is clear that with proper design, the smart spaces can be used for supporting user's wellbeing. Wellbeing can be seen from different perspectives: as a general phenomenon, or as an objective or subjective wellbeing of a person [9]. Subjective wellbeing of a person is connected into person's emotions which are usually triggered by an implicit cause (e.g., some event) [6]. One of the key impacting factors for peoples' subjective wellbeing is the living environment and as people spend half of their lives in work, it is crucial to support wellbeing at work too [9]. The WELL building standard [4] focuses on health and wellbeing of people occupying the buildings and it is aimed for all stakeholders involved in the construction and use of the buildings. Wellness in the space is affected by multiple factors and their interaction, most important factors being quality of air lighting, and comfort of people, fitness, nourishment, mind, and innovation [4].

To be able to anticipate users' actions and impact on their wellbeing in complex social circumstances, the system needs to be situation aware, e.g., understand human inner status and the current social situation. The sensor data collected from the environment might not be enough, therefore to some extent, wearable sensors could be used as they allow measuring the physiology of a human. For example, wellness bracelets or rings allow measuring user's heart rate variability [7] or the electrodermal activity (EDA) for indicating user's stress level [11]. Also, camera based facial recognition software (SW) is getting more and more mature and is able to distinguish facial expressions of the user $[8,13$ $16]$ which can be used in estimating people's emotions as each emotion is accompanied with a particular facial expression [6]. Understanding of peoples' perceptions and needs for this kind of systems in collaborative use situations is lacking. Therefore, we conducted an anticipated user experience (AUX) study with the concept video of adaptive meeting room supporting users' wellbeing. This paper presents the design challenges that rose from the study results.

\section{AUX Study}

User can anticipate her/his experience before the first use, during and after the actual use, and over time [10]. Pakanen [12] suggest studying AUX with visual design examples, therefore we created a concept video (Fig. $1 \& 2$ ) to investigate anticipated user experiences with the concept of an adaptive meeting room supporting users' wellbeing. The video describes an event taking place in a smart meeting room. The 
a)

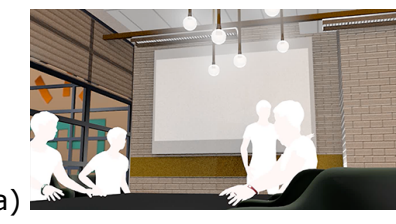

b)

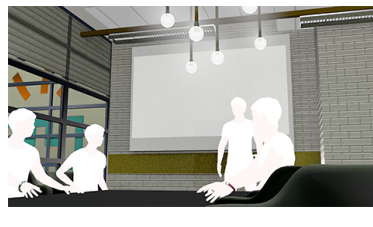

c)

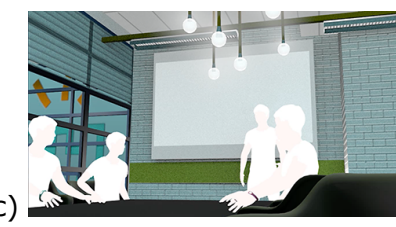

d)

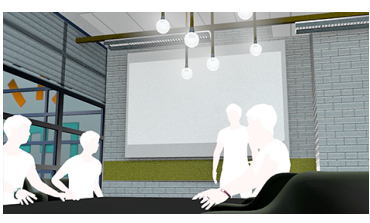

Figure 2: Lighting changes in the video $a, b$, $c$, and $d$.

a) People are irritated as one person arrives late to the meeting. To ensure easier new start of the meeting, system cools down the space and creates a more relaxing lighting by lowering the light output and making it warme to simulate the sunrise. system is presented in the left column box on page 2 (Fig. 1, a-b) and the main parts of the concept story on pages 3 and 4 with the lighting changes (Fig. 2, a-d).

Participants, Setting, Procedure, and Analysis We recruited 48 participants from the Eindhoven University of technology, TU/e, in The Netherlands (24) and University of Oulu in Finland (24). Before the evaluation participants filled a background questionnaire containing questions about demographics, use of wearable wellness tracking technology and applications, and attitudes towards and adoption of technology. Most of the participants were European (27) and Asian (18). The participants' ages varied from 23 to 51 with a mean of 33 and the gender distribution was 50/50. We identified 22 early adopters, 11 majority, and 15 late adopters. Wearable activity or fitness tracking devices were worn regularly by $19 / 48$.

Participants were familiarized to the study by a sensitizing video showing the day of the main character in the concept. Then, the main concept video was shown. Next, participants filled a semantic word pair questionnaire, applied from [1], with ten word pairs (Fig, 3). Then, a semi-structured interview was done.

The study resulted in total of 1200 minutes of audio recordings that were transcribed. Qualitative data analysis followed general qualitative coding principles [2]. Semantic word pair questionnaire results were analyzed with T-test between early and late adopters.

\section{Findings and Design Challenges}

Participants found the concept idea as interesting, innovative, novel, cool, and useful in certain use situations. The early adopters found the concept more positive than the late adopters in all semantic word pairs ( $p$ 0.0356) (Fig. 2). T-test showed significant differences between early and late adopters also in Pleasant-Unpleasant (p 0.0234) and Practical-

Impractical ( $p$ 0.0168) word pairs (Fig. 3).

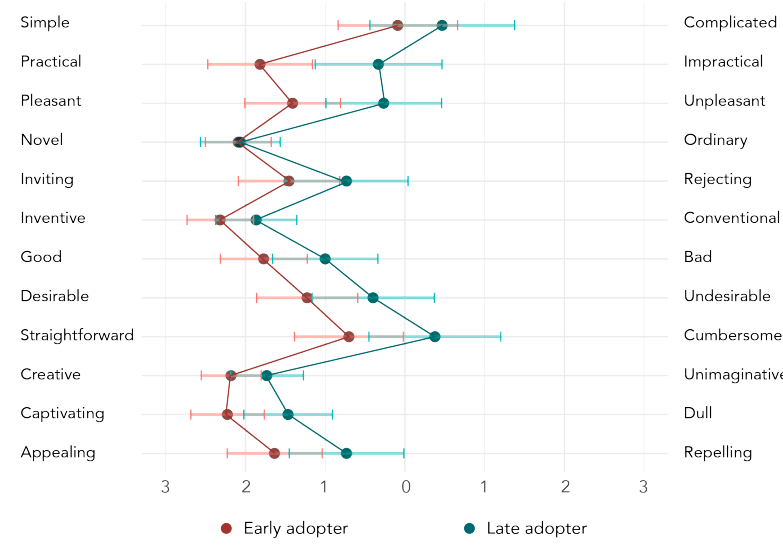

Figure 3: Semantic word pair questionnaire results indicating early adopters and late adopters' perceptions of the concept.

How we can make the environment suitable for all in collaborative use of the space? People were worried about if the system can consider individual differences and support several people in the smart space. For example, people sharing the room can have different facial expressions and stress levels, so how can the system adjust itself to provide a suitable lighting and temperature for all the users. Participants also stated that people have different preferences for temperature and lighting conditions. Therefore, the system that is based on average values might not be the best solution. Future research should study whether micro climates and individual lighting environments in a shared space can solve this problem. 
b) People in the room start to calm down and the meeting can carry on. After sensors and camera data confirm to the system that people are feeling more relaxed, it adjusts light output back to the cloudy day mode, a medium color temperature, and a light output which supports the PowerPoint presentations.

c) After half an hour of PowerPoint presentations, people start to look tired and are yawning. The system adjusts lights to match the color temperature with coole sunny daylight, and adjusts the light output to maximum to cheer up the people in the room.

d) After feeling more energized again, the presentation part ends and co-design phase starts. The facilitator sets the lighting in the room to "half cloudy daylight" mode which changes lighting atmosphere naturally, ensuring people stay alert and active throughout the ideation.
In what situations the climate and lighting changes are welcome? In general, the automatic adjustment of lights was perceived positively when the aim is to cheer up people, however indirect feedback that lighting changes could pose in certain situations was perceived as problematic. As one participant stated: "If you enter late to the meeting room, would this create a spotlighteffect...as the lighting changes so dramatically when you enter, so does it generate more stress?" (P29). Participants were also worried that lighting and aircondition speed changes during the presentation could indicate to the presenter that his/her presentation is boring as people need to be revitalized. In future, suitable situations should be further investigated.

System automation versus user control? Participants were both happy and annoyed about automatic adjustment of the room conditions. A preferred functionality would be that the system could change lighting and temperature, for example in the beginning of the meeting to make space more welcoming, to boost creativity and activity of the people in co-design and ideation sessions, and revitalize people periodically in long meetings. However, automatic adjustments were also perceived as patronizing: "I do not want technology to be my mom: Lesley honey go to bed... A should be the helper and not the decider of how you are going to live your life (P1). Another stated: "It's strange for a room to act as a mediator of emotions" (P12). People need to have control over the system especially, if its adjustments are not optimal. Future research should study how to inform users on what system is doing, e.g., to enhance peoples' awareness of system actions, and how to interact with the system.

How to assure that personal data is handled anonymously, it is used only for intended purpose only, and removed immediately after the analysis? The builtin sensors which measure $\mathrm{CO}^{2}$ and temperature levels in the room were perceived as essential for supporting wellbeing in the meeting room. However, wearable sensors and 360 degree camera with facial recognition SW were perceived more negatively as they collect personal data and an informed consent would be needed to approve the data collection. The biggest concern with wearable sensors is that they can tell more about the user than facial expression SW as you can show different facial expression than how you feel inside. As one participant stated: "People don't tell to other people what is going inside of them, but the [wearable] sensors tell" (P4). Therefore, people did not like sharing wearable sensor data with the room.

People were also concerned about what happens to the data that they share with the system. The problem is

that even though at the moment the collected data does not reveal too personal information, people acknowledged that in the future there might be more sophisticated algorithms and this 'innocent looking' heart rate data might turn against the space user. As participant stated: "You could do a history analysis algorithm and go back to that meeting and notice that certain guy was pissed and then they made that agreement [that impacted on our future]" (P41). Another concern was that the collected data will be used against the user, such as for evaluating performance of the worker and in worst case the worker might be laid off. Also people were worried that if data stays in the system, it could be hacked. It is a challenge for future research to solve the ethical and information security issues involved in using real-time data in this type of applications. The main goals are evoking the users' trust and gaining their consent. 


\section{Acknowledgements}

We thank all the study participants. This work has been funded by the Open Innovation Platform of Six City

Strategy project (A70202) funded by the ERDF and the City of Oulu and Business Finland with several companies through VIRPA-D project. The acquisition of the hardware for this study has also been partially funded by the ERDF project A71720, 'Big Data for 5G', governed by the HILLA program (www.hilla.center).

\section{References}

1. Attrakdiff. 2018. Retrieved July 9, 2018 from https://www.uid.com/en/publications/attrakdiff

2. Kathy Charmaz. 2008. Grounded theory as an emergent method. In Hesse-Bider S N \& Leavy P (eds) Handbook of Emergent Methods. New York NY, Guilford Press: 155-172.

3. Diane J. Cook \& Sajal K. Das. 2007. How smart are our environments? An updated look at the state of the art. Pervasive and mobile computing, 3(2), 5373.

4. Delos Living LLC. 2016. WELL Building Standard. Retrieved July 6, 2018 from https://tinyurl.com/y7c6j99y

5. Berry Eggen, Gerard Hollemans, \& Richard van de Sluis. 2003. Exploring and enhancing the home experience. Cognition, Technology \& Work, 5(1) 44-54.

6. Paul Ekman. 1992. An argument for basic emotions. Cognition and Emotion, 1992, 6 (3/4) 196-200.

7. Fitbit. 2018. Fitbit charge 2. Retrieved July 25, 2018 from https://www.fitbit.com/nl/charge2
8. Xiaohua Huang, Abhinav Dhall, Roland Goecke, Matti Pietikäinen, \& Guoying Zhao. 2018. Multimodel Framework for Analyzing the Affect of a Group of People. IEEE Transactions on Multimedia, 2018.

9. Felicia A. Huppert. 2014. The State of Wellbeing Science. In Wellbeing, C. L. Cooper (Ed.).

10. ISO 9241-210. 2010. Ergonomics of human-system interaction - Part 210: Human-centred design for interactive systems. Geneva: International Standardization Organization (ISO).

11. Moodmetric. 2016. The Moodmetric ring Retrieved July 6, 2018 from http://www.moodmetric.com

12. Minna Pakanen. 2015. Visual design examples in the evaluation of anticipated user experience at the early phases of research and development. Ph.D Dissertation. University of Oulu, Acta Univ. Oul. A 663.

13. Tomas Pfister, Matti Pietikäinen, Xiaobai Li, \& Guoying Zhao. 2014. Automated recognition algorithm for detecting facial expressions, US patent US 8848068 B2. Priority date: 2012-May08; Publication date: 2014-Sep-30.

14. Mahadev Satyanarayanan. 2001. Pervasive computing: Vision and challenges. IEEE Personal communications, 8(4), 10-17.

15. Mark Weiser. 1991. The Computer for the 21st Century. Scientific American, 265(3), 94-105.

16. Ligang Zhang, Brijesh Verma, Dian Tjondronegoro, and Vinod Chandran. 2018. Facial Expression Analysis under Partial Occlusion: A Survey. ACM Computing Surveys 51(2), Article 25. 